\title{
New normalized LMS adaptive filter with a variable regularization factor
}

\author{
LI Zhoufan, LI Dan* , XU Xinlong, and ZHANG Jianqiu \\ School of Information Science and Technology, Fudan University, Shanghai 200433, China
}

\begin{abstract}
A new normalized least mean square (NLMS) adaptive filter is first derived from a cost function, which incorporates the conventional one of the NLMS with a minimum-disturbance (MD) constraint. A variable regularization factor (RF) is then employed to control the contribution made by the MD constraint in the cost function. Analysis results show that the RF can be taken as a combination of the step size and regularization parameter in the conventional NLMS. This implies that these parameters can be jointly controlled by simply tuning the RF as the proposed algorithm does. It also demonstrates that the RF can accelerate the convergence rate of the proposed algorithm and its optimal value can be obtained by minimizing the squared noise-free posteriori error. A method for automatically determining the value of the RF is also presented, which is free of any prior knowledge of the noise. While simulation results verify the analytical ones, it is also illustrated that the performance of the proposed algorithm is superior to the state-of-art ones in both the steady-state misalignment and the convergence rate. A novel algorithm is proposed to solve some problems. Simulation results show the effectiveness of the proposed algorithm.
\end{abstract}

Keywords: adaptive filtering, normalized least mean square (NLMS), minimum-disturbance (MD) constraint, variable regularization, variable step-size NLMS.

DOI: $10.21629 / J S E E .2019 .02 .05$

\section{Introduction}

It is well known that the adaptive filter plays a very important role in many signal processing applications [1-5], e.g., the echo cancellation, line enhancement, channel equalization, system identification, and time delay estimation. In the past few decades, many adaptive filtering algorithms have been proposed for these applications. Among them, the least mean square (LMS) algorithm is a classical one, whose optimality and stability are fully discussed [6,7]. The normalized LMS (NLMS) algorithm

Manuscript received June 07, 2018.

*Corresponding author.

This work was supported by the National Natural Science Foundation of China $(61571131 ; 11604055)$. is an extension to the LMS for simplicity and robustness. However, the performances of both LMS and NLMS are governed by their step sizes, which reflect a tradeoff between the convergence rate and the misalignment.

In order to mitigate such a contradiction requirement, various variable step-size LMS (VSS-LMS) and -NLMS (VSS-NLMS) algorithms are proposed [8-26]. However, the step size used in most of these algorithms [8-19] is a function of the filtering error signal which is not equal to zero when the filter converges. This implies that such a nonzero step size will eventually lead to a nonzero misadjustment [1]. Moreover, these algorithms with the VSS could not work very reliably because their step sizes depend on either the characteristics of the noise or/and several parameters, which are hard to be tuned in practice.

In this paper, a new adaptive filter is first derived by introducing the minimum-disturbance (MD) constraint into the conventional cost function of the NLMS ones [1,2]. A variable regularization factor $\beta$ is then utilized to control the contribution made by the MD constraint. Analysis results show that the $\beta$ in our new algorithm merges the step size and the regularization parameter of the conventional NLMS into one variable. In this way, a more explicit physical description for these artificial parameters is given. A joint control strategy for them can be derived by simply tuning the regularization factor $\beta$ in the proposed algorithm. It also demonstrates that $\beta$ helps to reduce the instantaneous estimation errors of the second-order statistical moments and can thus accelerate the convergence rate of the algorithm. Analyses show that the optimal value of $\beta$ can be obtained by minimizing the squared noise-free posteriori error. A method free of any prior knowledge of the noise is then presented for updating $\beta$ automatically. While simulation results verify the analytical ones, it also illustrates that the proposed algorithm outperforms the stateof-art VSS-LMS and VSS-NLMS ones in both the steadystate misalignment and the convergence rate.

The rest of this paper is organized as follows. In Sec- 
tion 2 , the necessary background is introduced. The derivation of the proposed algorithm is given in Section 3. Section 4 analyzes the performance of the proposed algorithm and gives the method free of any prior knowledge of the noise for adjusting the regularization factor. Simulation results are reported in Section 5 and conclusions are drawn in Section 6.

\section{Background}

\subsection{System model}

In the system identification, the desired signal of an adaptive filter at the discrete-time instant $n$ is given as

$$
d(n)=y(n)+v(n)=\boldsymbol{w}^{\mathrm{T}}(n) \boldsymbol{x}(n)+v(n)
$$

where $y(n)$ is the output signal of the unknown system, the superscript $(\cdot)^{\mathrm{T}}$ denotes the transposition, $\boldsymbol{w}(n)=$ $\left[w_{0}(n), w_{1}(n), \ldots, w_{L-1}(n)\right]^{\mathrm{T}} \in \mathbf{R}^{L}$ refers to the impulse response of the unknown system with a length of $L$, $\boldsymbol{x}(n)=[x(n), x(n-1), \ldots, x(n-L+1)]^{\mathrm{T}} \in \mathbf{R}^{L}$ is a vector containing the $L$ most recent samples of the input signal, and $v(n)$ is the system noise which is assumed to be independent of $\boldsymbol{x}(n)$.

The adaptive filter is generally assumed to have the same structure as the unknown system. In this way, the priori and posterior error signals $e(n)$ and $\varepsilon(n)$ can be respectively expressed as

$$
\begin{gathered}
e(n)=d(n)-\widehat{\boldsymbol{w}}^{\mathrm{T}}(n-1) \boldsymbol{x}(n) \\
\varepsilon(n)=d(n)-\widehat{\boldsymbol{w}}^{\mathrm{T}}(n) \boldsymbol{x}(n)
\end{gathered}
$$

where $\widehat{\boldsymbol{w}}(n)$ denotes the filter coefficient vector at time instant $n$.

\subsection{Conventional LMS and NLMS}

To infer the impulse response of the unknown system defined in (1), a general method to minimize the mean squared error (MSE) is described by (2) $[1,2]$, which is given by

$$
\xi(n)=E\left[e^{2}(n)\right]
$$

where $E(\cdot)$ means the mathematical expectation. When the filter coefficients are constant, i.e. $\widehat{\boldsymbol{w}}(n)=\boldsymbol{w}$ for every $n \in \mathbf{N}$, one can rewrite (4) as follows:

$$
\xi=E\left[d^{2}(n)\right]-2 \boldsymbol{w}^{\mathrm{T}} \boldsymbol{r}_{\boldsymbol{x} \boldsymbol{d}}+\boldsymbol{w}^{\mathrm{T}} \boldsymbol{R}_{\boldsymbol{x}} \boldsymbol{w}
$$

where $\boldsymbol{r}_{\boldsymbol{x} \boldsymbol{d}}=E[d(n) \boldsymbol{x}(n)]$ and $\boldsymbol{R}_{\boldsymbol{x}}=E\left[\boldsymbol{x}(n) \boldsymbol{x}^{\mathrm{T}}(n)\right]$. Meanwhile, the famous Wiener solution can be obtained by setting the gradient vector of $(5)$ to zero [1,2]. However, the Wiener solution can hardly be acquired in practice because the second-order statistical moments in (5), i.e. $\boldsymbol{r}_{\boldsymbol{x} \boldsymbol{d}}$ and $\boldsymbol{R}_{\boldsymbol{x}}$, are generally unavailable.
The conventional LMS algorithm deals with such a dilemma by resorting to a stochastic gradient descent method, where the instantaneous values of $\boldsymbol{r}_{\boldsymbol{x} \boldsymbol{d}}$ and $\boldsymbol{R}_{\boldsymbol{x}}$ are used to estimate their statistical ones. In this way, the gradient estimation of (5) with respect to $\boldsymbol{w}$ can be expressed as follows:

$$
\widehat{\nabla}_{\boldsymbol{w}} \xi=-2 \widehat{\boldsymbol{r}}_{\boldsymbol{x} \boldsymbol{d}}+2 \widehat{\boldsymbol{R}}_{\boldsymbol{x}} \boldsymbol{w}
$$

where $\widehat{\boldsymbol{r}}_{\boldsymbol{x} \boldsymbol{d}} \triangleq d(n) \boldsymbol{x}(n)$ and $\widehat{\boldsymbol{R}}_{\boldsymbol{x}} \triangleq \boldsymbol{x}(n) \boldsymbol{x}^{\mathrm{T}}(n)$. Meanwhile, its coefficient vector can be updated as

$$
\begin{gathered}
\widehat{\boldsymbol{w}}(n)=\widehat{\boldsymbol{w}}(n-1)-\mu \widehat{\nabla}_{\boldsymbol{w}} \xi= \\
\widehat{\boldsymbol{w}}(n-1)+2 \mu e(n) \boldsymbol{x}(n)
\end{gathered}
$$

where $\mu$ is a positive scalar known as the step size which is generally fixed in the conventional LMS algorithms. However, the inherent limitation of a fixed $\mu$ necessitates the compromise between the conflicting requirements of the fast convergence speed and the small misadjustment [2].

An extension to the LMS algorithm, namely the NLMS, is obtained by normalizing the input signal by itself. Meanwhile, the update equation for the filter coefficients is given by

$$
\widehat{\boldsymbol{w}}(n)=\widehat{\boldsymbol{w}}(n-1)+\frac{2 \mu e(n) \boldsymbol{x}(n)}{\delta+\boldsymbol{x}^{\mathrm{T}}(n) \boldsymbol{x}(n)}
$$

where $\delta$ is a small positive number used to avoid the denominator in (8) being divided by zero. Although the NLMS algorithm solves the sensitivity of the LMS to the scaling of its input signal, it still fails to balance the convergence speed and the steady state misadjustment.

\section{New NLMS algorithm}

Let us assume that the coefficient vector of the adaptive filter in (1) is updated [17] in the following formation:

$$
\widehat{\boldsymbol{w}}(n)=\widehat{\boldsymbol{w}}(n-1)+\alpha(n) \boldsymbol{x}(n) .
$$

It can be seen from (9) that the filter coefficients are updated by the previous ones plus a weighted input signal. In this way, the aim of the adaptive filtering is to find a proper weight $\alpha(n)$ to make the filter converge to its true coefficients as fast as possible. To do so, the conventional NLMS algorithm employs the squared posteriori error as its cost function $[1,2]$, which is given by

$$
\begin{gathered}
\varepsilon^{2}(n)=d^{2}(n)-2 d(n) \boldsymbol{w}^{\mathrm{T}}(n) \boldsymbol{x}(n)+ \\
\boldsymbol{w}^{\mathrm{T}}(n) \boldsymbol{x}(n) \boldsymbol{x}^{\mathrm{T}}(n) \boldsymbol{w}(n)= \\
d^{2}(n)-2 \boldsymbol{w}^{\mathrm{T}}(n) \widehat{\boldsymbol{r}}_{\boldsymbol{x} \boldsymbol{d}}+\boldsymbol{w}^{\mathrm{T}}(n) \widehat{\boldsymbol{R}}_{\boldsymbol{x}} \boldsymbol{w}(n) .
\end{gathered}
$$

Meanwhile, the weight $\alpha(n)$ can be found by minimizing (10). After some straightforward mathematical manipulations, one has

$$
\alpha(n)=e(n)\left[\boldsymbol{x}^{\mathrm{T}}(n) \boldsymbol{x}(n)\right]^{-1} .
$$


To trade off the convergence speed and the misadjustment as well as avoid dividing by zero, a step size $\mu$ and regularization parameter $\delta$ are introduced as follows:

$$
\alpha(n)=2 \mu e(n)\left[\boldsymbol{x}^{\mathrm{T}}(n) \boldsymbol{x}(n)+\delta\right]^{-1} .
$$

The updated equation (8) for the NLMS algorithm can now be obtained by substituting (12) into (9). However, such an artificial step size $\mu$ and a regularization parameter $\delta$ will make the NLMS algorithm very difficult to use in practice. As a result, a lot of studies are focused on how to determine the value of $\mu$ and/or $\delta$, e.g., [8-16,19-30]. In this paper, these artificial parameters can be avoided by employing the following cost function, i.e.,

$$
\widetilde{\xi}(n)=\varepsilon^{2}(n)+\beta\|\widehat{\boldsymbol{w}}(n)-\widehat{\boldsymbol{w}}(n-1)\|^{2}
$$

where $\beta(\beta>0)$ is a variable regularization factor. The cost function (13) is composed of the squared posteriori error and the MD constraint [31] weighted by $\beta$. Note that we call $\beta$ and $\delta$ as the regularization factor and the parameter respectively in order to distinguish them. One can find that the regularization factor $\beta$ can control the contribution made by the MD constraint. To be specific, a small $\beta$ indicates that we care more about the squared a posteriori error as the conventional NLMS algorithm does in (10). Otherwise, the stationary filtering coefficients are more concerned. Note that, although a similar cost function is used [31], the effect of $\beta$ on the weight update and the method for determining it are unavailable. In the following part of this section and Section 4, we will answer the two questions.

To minimize (13), one can substitute (9) into it and calculate the derivative of $\widetilde{\xi}(n)$ on $\alpha(n)$ as follows:

$$
\begin{gathered}
\frac{\partial \widetilde{\xi}(n)}{\partial \alpha(n)}=2 \varepsilon(n) \frac{\partial \varepsilon(n)}{\partial \alpha(n)}+2 \beta \alpha(n) \boldsymbol{x}^{\mathrm{T}}(n) \boldsymbol{x}(n)= \\
2\left[d(n)-\widehat{\boldsymbol{w}}^{\mathrm{T}}(n-1) \boldsymbol{x}(n)-\alpha(n) \boldsymbol{x}^{\mathrm{T}}(n) \boldsymbol{x}(n)\right] \times \\
{\left[-\boldsymbol{x}^{\mathrm{T}}(n) \boldsymbol{x}(n)\right]+2 \beta \alpha(n) \boldsymbol{x}^{\mathrm{T}}(n) \boldsymbol{x}(n) .}
\end{gathered}
$$

Let $\partial \xi(n) / \partial \alpha(n)=0$, the weight $\alpha(n)$ can be obtained as

$$
\alpha(n)=\frac{d(n)-\widehat{\boldsymbol{w}}^{\mathrm{T}}(n-1) \boldsymbol{x}(n)}{\boldsymbol{x}^{\mathrm{T}}(n) \boldsymbol{x}(n)+\beta} .
$$

Substituting (15) into (9), we have

$$
\begin{aligned}
\widehat{\boldsymbol{w}}(n)= & \widehat{\boldsymbol{w}}(n-1)+\frac{d(n)-\widehat{\boldsymbol{w}}^{\mathrm{T}}(n-1) \boldsymbol{x}(n)}{\boldsymbol{x}^{\mathrm{T}}(n) \boldsymbol{x}(n)+\beta} \boldsymbol{x}(n)= \\
& \widehat{\boldsymbol{w}}(n-1)+\frac{1}{\boldsymbol{x}^{\mathrm{T}}(n) \boldsymbol{x}(n)+\beta} e(n) \boldsymbol{x}(n)
\end{aligned}
$$

which constitutes our new NLMS adaptive filtering algorithm with a variable regularization factor $\beta(\beta>0)$.
Comparing (16) with (8), following by some simple mathematical manipulations, it is easy for one to obtain

$$
\beta=\frac{1}{2 \mu}\left[\delta+(1-2 \mu) \boldsymbol{x}^{\mathrm{T}}(n) \boldsymbol{x}(n)\right] .
$$

It can be seen from (17) that the artificial parameters $\mu$ and $\delta$ in an NLMS algorithm are now merged into the new regularization factor $\beta$. It means that, by tuning $\beta$, we can equivalently adjust $\mu$ and $\delta$ simultaneously, which will thus greatly reduce the complexity of the control method for acquiring a low misadjustment with a fast convergence rate. Moreover, by means of $\beta$, an explicit physical description for the artificial parameters $\mu$ and $\delta$ is obtained, i.e., these two parameters are jointly used to control the contribution of the MD constraint in (13). In particular, let us assume $\delta=\theta \sigma_{x}^{2}$, where $\sigma_{x}^{2}=E\left[\boldsymbol{x}^{2}(n)\right]$ is the signal power, $\theta$ is a positive constant, and $\boldsymbol{x}^{\mathrm{T}}(n) \boldsymbol{x}(n) \approx L \sigma_{x}^{2}$, which holds for large values of $L$. Meanwhile, we have

$$
\beta \approx \sigma_{x}^{2}\left(\frac{L+\theta}{2 \mu}-L\right) .
$$

One can find from (18) that the step size $\mu$ is inversely proportional to the regularization factor $\beta$, and thus reversely controls the contribution of the MD constraint. In this way, another explanation for the control strategy of $\mu$ can be given as follows. A large step size suggests that less attention will be paid on the stationarity of the filtering coefficients, which is generally required in the initial state. On the other hand, a small step size, or, equivalently, a stationary filtering coefficient vector, is asked when the algorithm is approaching to its convergence. Such a conclusion exactly agrees with the ones in the VSS-NLMS algorithms. More details of the relationship between $\mu$ and $\beta$ will be discussed in next section.

\section{Performance analysis}

In this section, we will analyze our algorithm and discuss how $\beta$ influences the performance of the proposed algorithm. A method for determining $\beta$ automatically will also be presented.

\subsection{Convergence analysis}

\subsubsection{Promised convergence}

Lemma 1 The algorithm (16) converges to the optimal Wiener solution whatever the regularization factor $\beta>0$ is. The smaller the $\beta$ is, the faster the convergence is.

The proof of Lemma 1 is given at the end of this subsection. Lemma 1 tells us, when $\beta>0$, the convergence of our algorithm (16) is guaranteed. In contrast, the step size $\mu$ in the NLMS algorithm must be limited within a certain range, otherwise its convergence cannot be assured 
[1]. Such a result implies that our algorithm (16) is more flexible in the parameter setting or tuning.

We can also use the known results of the NLMS algorithm [1,2] to explain Lemma 1, i.e., within the range of convergence, the larger the step size $\mu$ is, the faster the convergence is. Recall the conclusion drawn from (18) that $\mu$ is inversely proportional to $\beta$. In this way, Lemma 1 actually reaches the same result as that of the NLMS algorithm.

Proof Take the mathematical expectation of (16), we have

$$
\begin{gathered}
E[\widehat{\boldsymbol{w}}(n)]=E[\widehat{\boldsymbol{w}}(n-1)]+ \\
E\left[\frac{\boldsymbol{x}(n) d(n)-\boldsymbol{x}^{\mathrm{T}}(n) \boldsymbol{x}(n) \widehat{\boldsymbol{w}}(n-1)}{\boldsymbol{x}^{\mathrm{T}}(n) \boldsymbol{x}(n)+\beta}\right] .
\end{gathered}
$$

For a large value of $L, \boldsymbol{x}^{\mathrm{T}}(n) \boldsymbol{x}(n) \approx L \sigma_{x}^{2}$ holds [15]. Assume that $\boldsymbol{w}(n)$ is independent of $\boldsymbol{x}(n)$, (19) can be rewritten as follows:

$$
\begin{gathered}
E[\widehat{\boldsymbol{w}}(n)]=E[\widehat{\boldsymbol{w}}(n-1)]+ \\
\frac{E[\boldsymbol{x}(n) d(n)]-E\left[\boldsymbol{x}(n) \boldsymbol{x}^{\mathrm{T}}(n)\right] E[\widehat{\boldsymbol{w}}(n-1)]}{L \sigma_{x}^{2}+\beta}= \\
E[\widehat{\boldsymbol{w}}(n-1)]+\frac{\boldsymbol{r}_{\boldsymbol{x} \boldsymbol{d}}-\boldsymbol{R}_{\boldsymbol{x}} E[\widehat{\boldsymbol{w}}(n-1)]}{L \sigma_{x}^{2}+\beta} .
\end{gathered}
$$

It is well known that the optimal solution of Wiener filtering [1] is

$$
\boldsymbol{w}_{\mathrm{opt}}=\boldsymbol{R}_{\boldsymbol{x}}^{-1} \boldsymbol{r}_{\boldsymbol{x} \boldsymbol{d}}
$$

Substituting (21) into (20), we get

$$
\begin{gathered}
E[\widehat{\boldsymbol{w}}(n)]-\boldsymbol{w}_{\mathrm{opt}}=\left[\boldsymbol{I}_{\boldsymbol{L}}-\frac{\boldsymbol{R}_{\boldsymbol{x}}}{L \sigma_{x}^{2}+\beta}\right] \times \\
\left\{E[\widehat{\boldsymbol{w}}(n-1)]-\boldsymbol{w}_{\mathrm{opt}}\right\} .
\end{gathered}
$$

Denoting $\boldsymbol{v}(n)=\widehat{\boldsymbol{w}}(n)-\boldsymbol{w}_{\text {opt }}$, we can rewrite (22) as

$$
E[\boldsymbol{v}(n)]=\left[\boldsymbol{I}_{\boldsymbol{L}}-\frac{\boldsymbol{R}_{\boldsymbol{x}}}{L \sigma_{x}^{2}+\beta}\right] E[\boldsymbol{v}(n-1)] .
$$

Decompose the matrix $\boldsymbol{R}_{\boldsymbol{x}}$ as

$$
\boldsymbol{R}_{\boldsymbol{x}}=\boldsymbol{Q} \Lambda Q^{-1}
$$

where $\boldsymbol{Q}$ is a unitary matrix, $\boldsymbol{\Lambda}=\operatorname{diag}\left(\lambda_{1}, \lambda_{2}, \ldots, \lambda_{L}\right)$ is a diagonal matrix with positive diagonal elements.

Denoting $\boldsymbol{v}(0)=\widehat{\boldsymbol{w}}(0)-\boldsymbol{w}_{\mathrm{opt}}$ as the initial value, and substituting (24) into (23), one has

$$
E[\boldsymbol{v}(n)]=\boldsymbol{Q}\left[\boldsymbol{I}_{\boldsymbol{L}}-\frac{\boldsymbol{\Lambda}}{L \sigma_{x}^{2}+\beta}\right]^{n} \boldsymbol{Q}^{-1} E[\boldsymbol{v}(0)] .
$$

Let

$$
\boldsymbol{v}^{\prime}(n)=\boldsymbol{Q}^{-1} \boldsymbol{v}(n) .
$$

Equation (25) can be rewritten as

$$
E\left[\boldsymbol{v}^{\prime}(n)\right]=\left[\boldsymbol{I}_{\boldsymbol{L}}-\frac{\boldsymbol{\Lambda}}{L \sigma_{x}^{2}+\beta}\right]^{n} E\left[\boldsymbol{v}^{\prime}(0)\right] .
$$

Recall that $\boldsymbol{R}_{\boldsymbol{x}}$ is positive $\left(\lambda_{i}>0, i=1, \ldots, L\right)$, and $\operatorname{tr}\left[\boldsymbol{R}_{\boldsymbol{x}}\right]=L \sigma_{x}^{2}=\sum \lambda_{i}$. In this way, we have

$$
0<1-\frac{\lambda_{i}}{L \sigma_{x}^{2}+\beta}<1, \quad i=1, \ldots, L .
$$

Based on (28), one can easily infer

$$
\lim _{n \rightarrow \infty}\left[\boldsymbol{I}_{\boldsymbol{L}}-\frac{\boldsymbol{\Lambda}}{L \sigma_{x}^{2}+\beta}\right]^{n}=0 .
$$

Insert (29) into (27) and take (26) into consideration, one has

Accordingly, we have

$$
\lim _{n \rightarrow \infty} E[\boldsymbol{v}(n)]=0 .
$$

$$
\lim _{n \rightarrow \infty} E[\widehat{\boldsymbol{w}}(n)]=\boldsymbol{w}_{\mathrm{opt}} .
$$

When $\beta>0$, it can be seen from (31) that the algorithm will converge to the optimal solution whatever the value of $\beta$ is. Moreover, one can find from (23) that the smaller the $\beta$ is, the faster the convergence is.

\subsubsection{Accelerated convergence rate}

When the new cost function (13) is minimized, it is well known that its constant positive factors can be neglected. As a result, we can rescale the right-hand side of (13) by $1 /(1+\widetilde{\beta})$, where $\widetilde{\beta}=\beta / \sigma_{x}^{2}$ is a rescaled regularization factor with a positive value. Because of the aforementioned reason, such a scale transformation will not influence the conclusions drawn before. Meanwhile, by substituting (16) into (13), our new cost function can be replaced by

$$
\widetilde{\xi}(n)=d^{2}(n)-2 \boldsymbol{w}^{\mathrm{T}}(n) \widetilde{\boldsymbol{r}}_{\boldsymbol{x} \boldsymbol{d}}+\boldsymbol{w}^{\mathrm{T}}(n) \widetilde{\boldsymbol{R}}_{\boldsymbol{x}} \boldsymbol{w}(n)
$$

where

$$
\begin{gathered}
\widetilde{\boldsymbol{r}}_{\boldsymbol{x} \boldsymbol{d}}=\frac{[d(n) \boldsymbol{x}(n)]+\widetilde{\beta}\left[\sigma_{x}^{2} \widehat{\boldsymbol{w}}(n-1)\right]}{1+\widetilde{\beta}}= \\
\frac{\widehat{\boldsymbol{r}}_{\boldsymbol{x} \boldsymbol{d}}+\widetilde{\beta}\left[\sigma_{x}^{2} \widehat{\boldsymbol{w}}(n-1)\right]}{1+\widetilde{\beta}} \\
\widetilde{\boldsymbol{R}}_{\boldsymbol{x}}=\frac{\left[\boldsymbol{x}^{\mathrm{T}}(n) \boldsymbol{x}(n)\right]+\widetilde{\beta}\left[\sigma_{x}^{2} \boldsymbol{I}_{\boldsymbol{L}}\right]}{1+\widetilde{\beta}}=\frac{\widehat{\boldsymbol{R}}_{\boldsymbol{x}}+\widetilde{\beta}\left[\sigma_{x}^{2} \boldsymbol{I}_{\boldsymbol{L}}\right]}{1+\widetilde{\beta}}
\end{gathered}
$$

stand for the equivalent statistic estimations of $\boldsymbol{r}_{\boldsymbol{x}}$ and $\boldsymbol{R}_{\boldsymbol{x}}$ in our algorithm respectively, and $\boldsymbol{I}_{\boldsymbol{L}}$ is a unit matrix with the size of $L \times L$. At this point, the following assumptions are made.

\section{Assumption 1}

(i) The autocorrelation matrix of the input is close to a diagonal one, i.e., $\boldsymbol{R}_{\boldsymbol{x}} \approx \sigma_{x}^{2} \boldsymbol{I}_{\boldsymbol{L}}$.

(ii) The unknown system is time-invariant, i.e., $\boldsymbol{w}(n)=$ $\boldsymbol{w}$ for every $n \in \mathbf{N}$. 
This assumption is widely used to simplify the analysis [23]. Moreover, it can be derived from Assumption 1 that $\boldsymbol{r}_{\boldsymbol{x} \boldsymbol{d}}=\sigma_{x}^{2} \boldsymbol{w}$, which has been verified in [33]. Meanwhile, the relationship between our statistic estimations, i.e., $\widetilde{\boldsymbol{r}}_{\boldsymbol{x} \boldsymbol{d}}$ and $\widetilde{\boldsymbol{R}}_{\boldsymbol{x}}$, and the instantaneous ones given in (6) can be established.

Lemma 2 Suppose that Assumption 1 holds. Let $\widehat{\boldsymbol{R}}_{\boldsymbol{x}}=\boldsymbol{R}_{\boldsymbol{x}}+\boldsymbol{\Delta}_{\boldsymbol{R}}$ and $\widehat{\boldsymbol{r}}_{\boldsymbol{x} \boldsymbol{d}}=\boldsymbol{r}_{\boldsymbol{x} \boldsymbol{d}}+\boldsymbol{\Delta}_{\boldsymbol{r}}$, where $\boldsymbol{\Delta}_{\boldsymbol{R}}$ and $\boldsymbol{\Delta}_{r}$ are the estimation errors of $\widehat{\boldsymbol{R}}_{\boldsymbol{x}}$ and $\widehat{\boldsymbol{r}}_{\boldsymbol{x} d}$, respectively. Then the following holds:

(i) $\widetilde{\boldsymbol{R}}_{\boldsymbol{x}}=\boldsymbol{R}_{\boldsymbol{x}}+\boldsymbol{\Delta}_{\boldsymbol{R}} /(1+\widetilde{\beta})$.

(ii) When $\widehat{\boldsymbol{w}}(n-1)$ is near the convergence, $\widetilde{\boldsymbol{r}}_{\boldsymbol{x} \boldsymbol{d}} \approx$ $\boldsymbol{r}_{\boldsymbol{x} \boldsymbol{d}}+\boldsymbol{\Delta}_{\boldsymbol{r}} /(1+\widetilde{\beta})$.

Proof According to Assumption 1, we have $\boldsymbol{R}_{\boldsymbol{x}} \approx$ $\sigma_{x}^{2} \boldsymbol{I}_{\boldsymbol{L}}$ and $\boldsymbol{r}_{\boldsymbol{x} \boldsymbol{d}}=\sigma_{x}^{2} \boldsymbol{w}$ [33]. In this way, Lemma 2 can be easily inferred from (33) and (34) with some straightforward mathematical manipulations.

Lemma 2 suggests that the estimation errors of $\widehat{\boldsymbol{R}}_{\boldsymbol{x}}$ and $\widehat{\boldsymbol{r}}_{\boldsymbol{x} \boldsymbol{d}}$ are shrunk by $1+\widetilde{\beta}$ in our algorithm, which makes $\widetilde{\boldsymbol{r}}_{x \boldsymbol{d}}$ and $\widetilde{\boldsymbol{R}}_{\boldsymbol{x}}$ close to their statistic true values. It means that our cost function (32) amounts to a closer approximation to (5) compared with the one given in (10) used by the conventional NLMS algorithm. Recall that our algorithm (16) and the conventional NLMS (8) are proved to be the optimal update equations for (10) and (32), respectively, and both of them are promised to converge to the Wiener solution (See Lemma 1 and [1,2]). As a result, one can expect that an accelerated convergence rate can be obtained by our algorithm compared with the conventional NLMS because our cost function is closer to (5).

\subsection{Misadjustment analysis}

Lemma 3 The misadjustment of the algorithm (16) can be adjusted by the regularization factor $\beta$, the larger the $\beta$ is, the lower the misadjustment is. When $\beta \rightarrow \infty$, the misadjustment will tend to zero.

\section{Proof}

Define the optimal instantaneous signal error as

$$
e_{\mathrm{opt}}(n)=d(n)-\boldsymbol{w}_{\mathrm{opt}}^{\mathrm{T}} \boldsymbol{x}(n)
$$

where $\boldsymbol{w}_{\text {opt }}$ denotes the optimal solution of Wiener filtering. Inserting (35) into (16) and denoting $\boldsymbol{v}(n)=\widehat{\boldsymbol{w}}(n)-$ $\boldsymbol{w}_{\text {opt }}$, we have

$$
\boldsymbol{v}(n)=\frac{\boldsymbol{v}(n-1)^{\mathrm{T}} \boldsymbol{x}(n)-e_{\mathrm{opt}}(n)}{\boldsymbol{x}^{\mathrm{T}}(n) \boldsymbol{x}(n)+\beta} \boldsymbol{x}(n) .
$$

For a large value of $L, \boldsymbol{x}^{\mathrm{T}}(n) \boldsymbol{x}(n) \approx L \sigma_{x}^{2}$ holds [15]. Meanwhile, we have

$$
\boldsymbol{v}(n)=\left[\boldsymbol{I}_{\boldsymbol{L}}-\frac{\boldsymbol{x}(n) \boldsymbol{x}^{\mathrm{T}}(n)}{L \sigma_{x}^{2}+\beta}\right] \boldsymbol{v}(n-1)+\frac{\boldsymbol{x}(n) e_{\mathrm{opt}}(n)}{L \sigma_{x}^{2}+\beta} .
$$

Assume $\boldsymbol{x}(n)$ is independent of $\boldsymbol{v}(n)$, and $e_{\mathrm{opt}}(n)$ is independent of $\boldsymbol{x}(n)$. Based on (37), we have

$$
\begin{gathered}
E\left[\boldsymbol{v}(n) \boldsymbol{v}^{\mathrm{T}}(n)\right]=E\left[\boldsymbol{v}(n-1) \boldsymbol{v}^{\mathrm{T}}(n-1)\right]- \\
2 \frac{\boldsymbol{R}_{\boldsymbol{x}}}{L \sigma_{x}^{2}+\beta} E\left[\boldsymbol{v}(n-1) \boldsymbol{v}^{\mathrm{T}}(n-1)\right]+ \\
\frac{\boldsymbol{R}_{\boldsymbol{x}}}{L \sigma_{x}^{2}+\beta} E\left[\boldsymbol{v}(n-1) \boldsymbol{v}^{\mathrm{T}}(n-1)\right] \frac{\boldsymbol{R}_{\boldsymbol{x}}}{L \sigma_{x}^{2}+\beta}+ \\
\frac{\boldsymbol{R}_{\boldsymbol{x}}}{\left(L \sigma_{x}^{2}+\beta\right)^{2}} \xi_{\text {min }}
\end{gathered}
$$

where $\xi_{\min }=E\left[\left|e_{\mathrm{opt}}(n)\right|^{2}\right]$ is the Wiener error [2].

For a large value of $L$, it is reasonable to assume $\boldsymbol{\Lambda} /\left(L \sigma_{x}^{2}+\beta\right) \ll \boldsymbol{I}$, where $\boldsymbol{R}_{\boldsymbol{x}}=\boldsymbol{Q} \boldsymbol{\Lambda} \boldsymbol{Q}^{-1}$ is the decomposition of the matrix $\boldsymbol{R}_{\boldsymbol{x}}$. As a result, the last term on the right-hand side of (38) can be neglected. From (38), one can derive

$$
\lim _{n \rightarrow \infty} E\left[\boldsymbol{v}(n) \boldsymbol{v}^{\mathrm{T}}(n)\right]=\frac{\xi_{\min }}{2\left(L \sigma_{x}^{2}+\beta\right)} \boldsymbol{I}_{\boldsymbol{L}} .
$$

From (39), one can conclude that the misadjustment of the algorithm will tend to zero as $\beta \rightarrow \infty$.

For the convenience of the following discussions, we here rewrite the misadjustment (38) as follows:

$$
E\left\{\|\widehat{\boldsymbol{w}}(n)-\boldsymbol{w}(n)\|^{2}\right\}=\frac{L}{2\left[L \sigma_{x}^{2}+\beta\right]} \xi_{\min } .
$$

One can obviously see from (40) that the misadjustment converges to zero when $\beta \rightarrow \infty$. We can also use the known results of the NLMS algorithm [1,2] to explain Lemma 3, i.e., within the range of the convergence, the smaller the step size $\mu$ is, the lower the misadjustment is. With the relationship given in (18) taken into consideration, one can find that Lemma 3 gets the same result as that of the NLMS algorithm again.

\subsection{Method for determining $\beta$}

Ideally, both a fast convergence and a low steady state misadjustment are required for a satisfactory performance in all conditions. However, Lemma 1 and Lemma 3 indicate that, although the value of $\beta$ does not change the convergence results of algorithm (16), it will affect the convergence rate and misadjustment. In the start stage of filtering or system change phases, the filter with a fast tracking is preferred. As a result, a small $\beta$ should be taken as Lemma 1 suggests. When the system is deeply converged, a large $\beta$ should be taken to make the misadjustment as low as possible. Ideally, when $\beta \rightarrow \infty$, the misadjustment will tend to zero as given in (40). To solve this seeming contradiction, we will discuss how to determine the value of $\beta$ in this subsection. 
Let us rewrite the priori error signal $e(n)$ as

$$
e(n)=e_{f}(n)+v(n)
$$

where

$$
e_{f}(n)=\boldsymbol{w}(n)^{\mathrm{T}} \boldsymbol{x}(n)-\widehat{\boldsymbol{w}}(n-1)^{\mathrm{T}} \boldsymbol{x}(n)
$$

is the noise-free priori error signal. Similarly, the posteriori error signal can be expressed as

$$
\varepsilon(n)=\varepsilon_{f}(n)+v(n)
$$

where

$$
\varepsilon_{f}(n)=\boldsymbol{w}(n)^{\mathrm{T}} \boldsymbol{x}(n)-\widehat{\boldsymbol{w}}(n)^{\mathrm{T}} \boldsymbol{x}(n)
$$

is the noise-free posteriori error signal. An optimal value for the regularization factor $\beta$ can be achieved by minimizing the expectation of $\varepsilon_{f}^{2}(n)$ [32]. To solve such a minimization problem, we can generalize (16) as

$$
\boldsymbol{w}(n)=\boldsymbol{w}(n-1)+\frac{q e(n) \boldsymbol{x}(n)}{\boldsymbol{x}^{\mathrm{T}}(n) \boldsymbol{x}(n)+\beta}
$$

where $q$ is an auxiliary variable which equals to one in our algorithm as shown in (16). By substituting (42) and (45) into (44), one has

$$
\varepsilon_{f}(n)=[1-q \tau(n)] e_{f}(n)-q \tau(n) v(n)
$$

where

$$
\begin{gathered}
\tau(n)=\frac{\rho(n)}{\beta+\rho(n)} \\
\rho(n)=\boldsymbol{x}(n)^{\mathrm{T}} \boldsymbol{x}(n) .
\end{gathered}
$$

By squaring both sides of (46) and taking the expectation, we have

$$
\begin{aligned}
& E\left[\varepsilon_{f}^{2}(n)\right]=E\left[e_{f}^{2}(n)\right]-2 q E\left[\tau(n) e_{f}^{2}(n)\right]+ \\
& q^{2} E\left[\tau^{2}(n) e_{f}^{2}(n)\right]+q^{2} E\left[\tau^{2}(n)\right] E\left[v^{2}(n)\right]
\end{aligned}
$$

To minimize (49), we can make its derivation with respect to $q$, that is

$$
\begin{gathered}
\frac{\partial E\left[\varepsilon_{f}^{2}(n)\right]}{\partial q}=-2 E\left[\tau(n) e_{f}^{2}(n)\right]+2 q E\left[\tau^{2}(n) e_{f}^{2}(n)\right]+ \\
2 q E\left[\tau^{2}(n)\right] E\left[v^{2}(n)\right]
\end{gathered}
$$

equals to zero. After some straightforward mathematical manipulations, we obtain

$$
q=\frac{E\left[\tau(n) e_{f}^{2}(n)\right]}{E\left[\tau^{2}(n) e_{f}^{2}(n)\right]+E\left[\tau^{2}(n)\right] E\left[v^{2}(n)\right]} .
$$

When the correlation between $\tau(n)$ and $e_{f}(n)$ is neglected [32], (51) can be approximated as follows:

$$
q \approx \frac{E[\tau(n)] \sigma_{f}^{2}(n)}{E\left[\tau^{2}(n)\right] \sigma_{f}^{2}(n)+E\left[\tau^{2}(n)\right] \sigma_{v}^{2}}
$$

where $\sigma_{f}^{2}(n)=E\left[e_{f}^{2}(n)\right]$ is the excess MSE (EMSE), and $\sigma_{v}^{2}=E\left[v^{2}(n)\right]$ denotes the system noise variance. Note that the EMSE is a function of time $n$ because the filter coefficients $\widehat{\boldsymbol{w}}(n-1)$ varies with $n$. We will then discuss the value of $\beta$ according to the two different conditions.

When $\beta \ll \rho(n)$, we have

$$
\tau(n)=\frac{\rho(n)}{\rho(n)+\beta}=1-\frac{\beta}{\rho(n)}+\left[\frac{\beta}{\rho(n)}\right]^{2}-\cdots .
$$

Neglect the higher order terms in (53), one has

$$
\tau(n) \approx 1-\frac{\beta}{\rho(n)} .
$$

According to (54), we can obtain

$$
E\left[\tau^{2}(n)\right]=1-2 \beta E\left[\frac{1}{\rho(n)}\right] .
$$

By neglecting the second-order term. On the other hand, it has been shown that $\rho(n) \approx L \sigma_{x}^{2}$ for a large value of $L$ [15]. As a result, $E\left[1 / \rho^{2}(n)\right]$ will tend to zero in this case. Meanwhile, taking (54) into consideration, one has

$$
E^{2}[\tau(n)]=\left\{E\left[1-\frac{\beta}{\rho(n)}\right]\right\}^{2} \approx 1-2 \beta E\left[\frac{1}{\rho(n)}\right] .
$$

Substitute (55) and (56) into (52), and let $q=1$, we have

$$
\beta=\frac{\sigma_{v}^{2}}{\sigma_{f}^{2}(n)+\sigma_{v}^{2}} L \sigma_{x}^{2}, \quad \beta \ll \rho(n) .
$$

If $\beta \gg \rho(n)$ holds, we have

$$
\tau(n)=\frac{\rho(n)}{\rho(n)+\beta} \approx \frac{\rho(n)}{\beta} .
$$

Similarly, let us assume $\rho(n) \approx L \sigma_{x}^{2}$ holds for a large value of $L$. In this way, substituting (58) into (52) and, again, making $q=1$, one has

$$
\beta=\frac{\sigma_{f}^{2}(n)+\sigma_{v}^{2}}{\sigma_{f}^{2}(n)} L \sigma_{x}^{2}, \quad \beta \gg \rho(n) .
$$

Combining (57) with (59), one can conclude that

$$
\beta=\left\{\begin{array}{ll}
\frac{\sigma_{v}^{2}}{\sigma_{f}^{2}(n)+\sigma_{v}^{2}} L \sigma_{x}^{2}, & \beta \ll \rho(n) \\
\frac{\sigma_{f}^{2}(n)+\sigma_{v}^{2}}{\sigma_{f}^{2}(n)} L \sigma_{x}^{2}, & \beta \gg \rho(n)
\end{array} .\right.
$$


A generalized formation of $\beta$ can then be inferred from (60) given by

$$
\beta=f\left[\sigma_{f}^{2}(n), \sigma_{v}^{2}\right] L \sigma_{x}^{2}
$$

where $f(\cdot): \mathbf{R} \rightarrow \mathbf{R}$ in which $\mathbf{R}$ means the real number field. Recall that $\rho(n) \approx L \sigma_{x}^{2}$ holds for a large value of $L$ [15]. In this way, by substituting (61) into (47), we have

$$
\tau(n)=\frac{1}{f\left[\sigma_{f}^{2}(n), \sigma_{v}^{2}\right]+1} .
$$

At this point, it is clear that the expectations of $\tau(n)$ and $\tau^{2}(n)$ can be calculated directly. By substituting (62) into (52) and taking $q=1$ into consideration, we have

$$
f\left[\sigma_{f}^{2}(n), \sigma_{v}^{2}\right]=\frac{\sigma_{v}^{2}}{\sigma_{f}^{2}(n)}
$$

and accordingly

$$
\beta=\frac{\sigma_{v}^{2}}{\sigma_{f}^{2}(n)} L \sigma_{x}^{2}
$$

It can be seen from (63) that, since $\rho(n) \approx L \sigma_{x}^{2}$ for a large $L, \sigma_{f}^{2}(n) \gg \sigma_{v}^{2}$ holds if $\beta \ll \rho(n)$. Meanwhile, by developing (60), we obtain $L \sigma_{v}^{2} \sigma_{x}^{2} /\left(\sigma_{f}^{2}(n)+\sigma_{v}^{2}\right) \approx$ $L \sigma_{v}^{2} \sigma_{x}^{2} / \sigma_{f}^{2}(n)$, which reaches the same result as (63). Similarly, we have $\sigma_{f}^{2}(n) \ll \sigma_{v}^{2}$ if $\beta \gg \rho(n)$. Under this condition, it can be derived from (59) that $L\left(\sigma_{f}^{2}(n)+\right.$ $\left.\sigma_{v}^{2}\right) \sigma_{x}^{2} / \sigma_{f}^{2}(n) \approx L \sigma_{v}^{2} \sigma_{x}^{2} / \sigma_{f}^{2}(n)$ which agrees with (63) again.

It is worth noting that $\sigma_{f}^{2}(n) \gg \sigma_{v}^{2}$ generally holds in the initial state or when a system change occurs. It means that, by means of (63), a small value of $\beta$ is obtained in this condition. Such a choice for $\beta$ exactly follows the conclusion drawn in Lemma 1, and thus results in a fast convergence rate. On the other hand, one has $\sigma_{f}^{2}(n) \ll \sigma_{v}^{2}$ in the steady state, which leads to a large $\beta$ as shown in (63). In this case, a small misadjustment can be obtained as Lemma 3 suggests, which, again, agrees with the discussion before.

Let us now assume $\boldsymbol{w}(n)=\boldsymbol{w}(n-1)+\boldsymbol{\mu}(n)$, where $\boldsymbol{\mu}(n)$ is a zero-mean Gaussian white noise vector with an independent identical distribution $N\left(0, \sigma_{\mu}^{2}\right)$. Meanwhile, by substituting (63) and $\rho(n) \approx L \sigma_{x}^{2}$ into (16), we have

$$
\begin{gathered}
\widehat{\boldsymbol{w}}(n)=\widehat{\boldsymbol{w}}(n-1)+ \\
\frac{\sigma_{f}^{2}(n)}{L \sigma_{x}^{2} \sigma_{f}^{2}(n)+L \sigma_{x}^{2} \sigma_{v}^{2}} \boldsymbol{x}(n) e(n) .
\end{gathered}
$$

For a large value of $L$ and $m(n) \triangleq E\left[\|\boldsymbol{w}(n)-\widehat{\boldsymbol{w}}(n)\|^{2}\right]$, where $\|\cdot\|$ is the Euclidean norm, we have

$$
e_{f}(n)=[\boldsymbol{w}(n)-\widehat{\boldsymbol{w}}(n-1)]^{\mathrm{T}} \boldsymbol{x}(n)=
$$

$$
[\boldsymbol{\delta}(n-1)+\boldsymbol{\mu}(n)]^{\mathrm{T}} \boldsymbol{x}(n)
$$

where $\boldsymbol{\delta}(n)=\boldsymbol{w}(n)-\widehat{\boldsymbol{w}}(n)$. Taking the mathematical expectation of the $l_{2}$-norm of (66) and defining $\psi(n)=$ $\boldsymbol{\delta}(n-1)+\boldsymbol{\mu}(n)$, we obtain

$$
\begin{gathered}
E\left[e_{f}^{2}(n)\right]=E\left\{\boldsymbol{\psi}^{\mathrm{T}}(n) \boldsymbol{x}(n) \boldsymbol{x}^{\mathrm{T}}(n) \boldsymbol{\psi}(n)\right\}= \\
E\left\{\operatorname{tr}\left[\boldsymbol{\psi}(n) \boldsymbol{\psi}^{\mathrm{T}}(n) \boldsymbol{x}(n) \boldsymbol{x}^{\mathrm{T}}(n)\right]\right\} .
\end{gathered}
$$

Let us now assume $\boldsymbol{x}(n), \boldsymbol{\delta}(n)$, and $\boldsymbol{\mu}(n)$ are independent with each other, and $E\left[\boldsymbol{\delta}(n) \boldsymbol{\delta}^{\mathrm{T}}(n)\right]$ is close to a diagonal matrix which is reasonable in a steady state [30]. Meanwhile, (67) becomes

$$
\begin{gathered}
E\left[e_{f}^{2}(n)\right]=\operatorname{tr}\left\{E\left[\boldsymbol{\psi}(n) \boldsymbol{\psi}^{\mathrm{T}}(n)\right] \times E\left[\boldsymbol{x}(n) \boldsymbol{x}^{\mathrm{T}}(n)\right]\right\}= \\
\operatorname{tr}\left\{\left[E\left[\boldsymbol{\delta}(n-1) \boldsymbol{\delta}^{\mathrm{T}}(n-1)\right]+\right.\right. \\
\left.\left.E\left[\boldsymbol{\mu}(n) \boldsymbol{\mu}^{\mathrm{T}}(n)\right]\right] E\left[\boldsymbol{x}(n) \boldsymbol{x}^{\mathrm{T}}(n)\right]\right\}= \\
\sigma_{x}^{2}\left\{E\left[\|\boldsymbol{\delta}(n-1)\|^{2}\right]+L \sigma_{\mu}^{2}\right\}= \\
\sigma_{x}^{2}\left[m(n-1)+L \sigma_{\mu}^{2}\right]
\end{gathered}
$$

By substituting (68) into (64), one has

$$
\begin{gathered}
\widehat{\boldsymbol{w}}(n)=\widehat{\boldsymbol{w}}(n-1)+ \\
\frac{\left[m(n-1)+L \sigma_{\mu}^{2}\right] \boldsymbol{x}(n) e(n)}{L \sigma_{v}^{2}+L \sigma_{x}^{2}\left[m(n-1)+L \sigma_{\mu}^{2}\right]} .
\end{gathered}
$$

On the other hand, the optimal update equation for the NLMS algorithm (8) is proposed [30], which is given by

$$
\begin{gathered}
\widehat{\boldsymbol{w}}(n)=\widehat{\boldsymbol{w}}(n-1)+ \\
\frac{\left[m(n-1)+L \sigma_{\mu}^{2}\right] \boldsymbol{x}(n) e(n)}{L \sigma_{v}^{2}+(L+2) \sigma_{x}^{2}\left[m(n-1)+L \sigma_{\mu}^{2}\right]} .
\end{gathered}
$$

Comparing (69) with (70), one can find that they nearly agree with each other for a large $L$. Although (69) in this paper and the one in [30] seem the same, they are derived from different ways. In this paper, (69) is acquired by tuning the value of $\beta$, which is used to control the contribution of the MD constraint in (13). However, [30] does so by directly searching for the joint optimal values for the step-size $\mu$ and the regularization parameter $\delta$, while the physical description for these optimal values remains unknown. Moreover, since there is an inherent relationship between the optimal $\beta$ and different requirements as discussed before, (63) can be taken as a measurement of the convergence depth. According to this measurement, the optimal extent, to which the statistic estimations $\widetilde{\boldsymbol{r}}_{\boldsymbol{x} \boldsymbol{d}}$ and $\widetilde{\boldsymbol{R}}_{\boldsymbol{x}}$ are pushed towards $\sigma_{x}^{2} \widehat{\boldsymbol{w}}(n-1)$ and $\sigma_{x}^{2} \boldsymbol{I}_{\boldsymbol{L}}$ respectively, is determined as shown in (33) and (34). It shows that their statistic estimation errors are greatly shrunk as 
Lemma 2 states. Such an effect constituted the main reason why function (69) lead to a satisfactory performance, which was also absent in [30].

Clearly, (63) is fairly hard to use because $\sigma_{f}^{2}(n)$ is always unavailable in practice. A method for estimating $\sigma_{f}^{2}(n)$ was proposed in [30], however, the noise variance $\sigma_{v}^{2}$ was still required. In the rest of this subsection, we will discuss how to determine the value of $\beta$ without any prior knowledge.

If Assumption 1 (ii) is satisfied, then we have $\boldsymbol{w}_{\mathrm{opt}}=$ $\boldsymbol{w}$, where $\boldsymbol{w}_{\text {opt }}=\boldsymbol{R}_{\boldsymbol{x}}^{-1} \boldsymbol{r}_{\boldsymbol{x} \boldsymbol{d}}$ denotes the optimal solution of Wiener filtering [1]. In this way, $\sigma_{f}^{2}$ can be expresses as follows:

$$
\begin{gathered}
\sigma_{f}^{2}(n)=E\left[e_{f}^{2}(n)\right]= \\
E\left[\boldsymbol{v}^{\mathrm{T}}(n-1) \boldsymbol{x}(n) \boldsymbol{x}^{\mathrm{T}}(n) \boldsymbol{v}(n-1)\right]= \\
E\left\{\operatorname{tr}\left[\boldsymbol{v}(n-1) \boldsymbol{v}^{\mathrm{T}}(n-1) \boldsymbol{x}(n) \boldsymbol{x}^{\mathrm{T}}(n)\right]\right\}= \\
\operatorname{tr}\left\{E\left[\boldsymbol{v}(n-1) \boldsymbol{v}^{\mathrm{T}}(n-1) \boldsymbol{x}(n) \boldsymbol{x}^{\mathrm{T}}(n)\right]\right\}
\end{gathered}
$$

where $\boldsymbol{v}(n)=\widehat{\boldsymbol{w}}(n)-\boldsymbol{w}_{\mathrm{opt}}$ is the instantaneous coefficient error. Assume that $\boldsymbol{v}(n-1)$ is uncorrelated with $\boldsymbol{x}(n)$, one can rewrite (71) as follows:

$$
\sigma_{f}^{2}(n)=\sigma_{x}^{2} E\left[\boldsymbol{v}^{\mathrm{T}}(n-1) \boldsymbol{v}(n-1)\right] .
$$

Substitute (37) into (72) and let Assumption 1 (i) hold, we have

$$
\begin{gathered}
\sigma_{f}^{2}(n)=\left[1-\frac{\sigma_{x}^{2}}{L \sigma_{x}^{2}+\beta}\right]^{2} \sigma_{f}^{2}(n-1)+ \\
{\left[\frac{1}{L \sigma_{x}^{2}+\beta}\right]^{2} \sigma_{x}^{2} E\left[v^{2}(n) \boldsymbol{x}^{\mathrm{T}}(n) \boldsymbol{x}(n)\right] .}
\end{gathered}
$$

For a large value of $L$, the terms with a factor of $\left(L \sigma_{x}^{2}+\right.$ $\beta)^{-2}$ can be all neglected. Therefore, it results in

$$
\sigma_{f}^{2}(n) \approx\left[1-2 t \frac{\sigma_{x}^{2}}{L \sigma_{x}^{2}+\beta}\right] \sigma_{f}^{2}(n-1)
$$

where $t$ is a tuning factor $(0<t \leqslant 1)$ for compensating the assumptions we made before. Meanwhile, it can be seen from (63) that the regularization factor $\beta$ can be updated recursively according to (74), that is

$$
\beta(n)=\left[\frac{L \sigma_{x}^{2}+\beta(n-1)}{(L-2 t) \sigma_{x}^{2}+\beta(n-1)}\right] \beta(n-1) .
$$

One can find from (75) that $\beta(n)>\beta(n-1)$ for every $n \in \mathbf{N}$, which will thus lead to a misadjustment tending to zero as shown in Lemma 3.

Finally, we discuss two issues based on some practical considerations. The first one is how to obtain the input signal power $\sigma_{x}^{2}$ in (75). Fortunately, one can easily evaluate it [16] by

$$
\widehat{\sigma}_{x}^{2}(n)=\lambda \widehat{\sigma}_{x}^{2}(n-1)+(1-\lambda)_{x}^{2}(n)
$$

where $\lambda=1-1 /(L K)$ with an integral constant $K \geqslant 2$.

The second but more important issue is that (75) is based on Assumption 1 (ii), which, as a result, has not given a criterion to find whether a system change occurs. However, such a criterion is a common requirement in practice. To circumvent this problem, the change rate of the instantaneous prior error signal power is employed to detect the system changes, which is given by

$$
\varsigma(n)=\left|\frac{\widehat{\sigma}_{e}^{2}(n)-\widehat{\sigma}_{e}^{2}(n-L)}{\widehat{\sigma}_{e}^{2}(n-L)}\right|
$$

where $\widehat{\sigma}_{e}^{2}(n)$ stands for the power estimation of (2) at time instant $n$. Similar to (76), $\widehat{\sigma}_{e}^{2}(n)$ can be obtained by

$$
\widehat{\sigma}_{e}^{2}(n)=\lambda \widehat{\sigma}_{e}^{2}(n-1)+(1-\lambda)_{e}^{2}(n) .
$$

Based on (77), $\beta$ can be determined as follows. When $\varsigma(n)$ is larger than a threshold, it means that a system change is detected or the adaptive filter is in its initial state. Meanwhile, we have $\sigma_{f}^{2}(n) \gg \sigma_{v}^{2}$, which means that a small $\beta$ should be assigned according to (63). To be specific, $\beta=20 \sigma_{x}^{2}$ is also a common rule $[15,18,20,30]$. From Lemma 1, one can also find that a fast convergence rate can then be obtained because $\beta$ exactly meets our requirements. Otherwise, when $\varsigma(n)$ is small, a stable system is implied and thus $\beta$ can be updated according to (75). In this way, a low misadjustment tending to zero is obtained as discussed before.

Based on the aforementioned discussions, the method for determining $\beta$ is summarized as follows:

$$
\text { If } \varsigma(n) \geqslant \frac{2}{K}
$$

$$
\beta(n)=20 \widehat{\sigma}_{x}^{2}(n)
$$

Else

$$
\beta(n)=\left[\frac{L \widehat{\sigma}_{x}^{2}(n)+\beta(n-1)}{(L-2 t) \widehat{\sigma}_{x}^{2}(n)+\beta(n-1)}\right] \beta(n-1) .
$$

It can be seen that our method is free of any prior knowledge of the system observation noise, and is thus much more practical than the ones reported in literature.

\section{Simulations and results analysis}

The performance of the proposed algorithm is evaluated in the context of system identification. The system to be identified is an impulse response of length $L=128$, where an abrupt change of the system is introduced at the 50 000th iteration. Its output $\boldsymbol{y}(n)$ is corrupted by a white Gaussian noise with a signal-to-noise ratio (SNR) of $20 \mathrm{~dB}$. The input $\boldsymbol{x}(n)$ is either a white Gaussian noise or an $\mathrm{AR}(1)$ process generated by filtering a white Gaussian noise through a first-order system $1 /\left(1-0.6 z^{-1}\right)$. The performance is 
evaluated in terms of the normalized misalignment $(\mathrm{dB})$ defined as $20 \log _{10}\left(\|\boldsymbol{w}(n)-\widehat{\boldsymbol{w}}(n)\|_{2} /\|\boldsymbol{w}(n)\|_{2}\right)$, and the results are averaged over 100 independent trials if not specified.

In the simulations, we compare the proposed algorithm with the conventional NLMS algorithm, four VSS-NLMS algorithms [13,15,16,30], and two VSS-LMS algorithms [26,29]. The parameter settings are tabulated in Table 1. Among these competitors, the noise power $\sigma_{v}^{2}$ was required in $[15,30]$, which was assumed to be exactly known as a priori to obtain their best performance. A benchmark, denoted as the miracle proposed algorithm, is also conceived by using (63) to determine $\beta$, where $\sigma_{v}^{2}, \sigma_{x}^{2}$, and $\sigma_{f}^{2}(n)$ are all known as a priori. According to (69) and (70), one can find that such an algorithm can also be taken as the benchmark of the JO-NLMS algorithm [30]. Note that the miracle proposed algorithm is impractical, because $\sigma_{f}^{2}(n)$ is unavailable in practice.

Table 1 Simulation parameters of the proposed algorithm and its competitors

\begin{tabular}{|c|c|c|}
\hline Algorithm & Variable step & Parameter \\
\hline SM-NLMS [13] & $\mu(n)=\left\{\begin{array}{l}1-\frac{\gamma}{|e(n)|}, \quad|e(n)|>\gamma \\
0, \text { else }\end{array}\right.$ & $\gamma=0.04 \ldots$ \\
\hline NPVSS-NLMS [15] & $\begin{array}{r}\mu(n)=\left\{\begin{array}{l}1-\frac{\sigma_{v}}{\widehat{\sigma}_{e}(n)+\varepsilon}, \quad \widehat{\sigma}_{e}(n) \geqslant \widehat{\sigma}_{e}(n) \\
0, \text { else }\end{array}\right. \\
\widehat{\sigma}_{e}(n)=\lambda \widehat{\sigma}_{e}(n-1)+(1-\lambda) e^{2}(n)\end{array}$ & $\begin{array}{l}\lambda=1-1 /(L K), \delta=20 \sigma_{x}^{2}, \varepsilon=10^{-3}, K=2 \\
\text { for a white input and } K=6 \text { for an } \mathrm{AR}(1) \text { process. } \\
\sigma_{v}^{2} \text { and } \sigma_{x}^{2} \text { are known as the priori. }\end{array}$ \\
\hline NVSS-NLMS [16] & 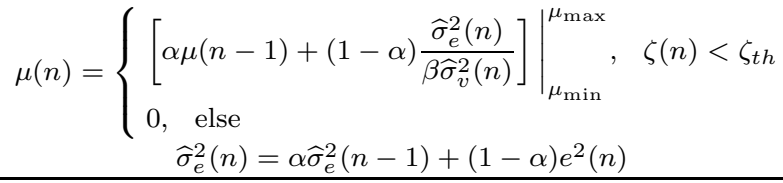 & $\begin{array}{l}\alpha=0.998, \beta=30, \zeta_{\mathrm{th}}=0.35, \mu_{\min }=10^{-5} \\
\text { and } \mu_{\max }=1\end{array}$ \\
\hline JO-NLMS [30] & $q(n)=\frac{p(n)}{L \sigma_{v}^{2}+(L+2) p(n) \widehat{\sigma}_{x}^{2}}$ & $\sigma_{v}^{2}$ is known as a priori. \\
\hline NVSS-LMS [26] & $\mu(n)=\beta\{1 /[1+\exp (-\alpha|e(n) e(n-1)|)]-0.5\}$ & $\alpha=20, \beta=0.01$ \\
\hline$l_{2}$-VSS-LMS [29] & $\begin{array}{c}\mu(n)=\beta\left[1-\exp \left(-\alpha|e(n)| \times\|\boldsymbol{P}(n)\|^{2}\right)\right] \\
\boldsymbol{P}(n)=a \boldsymbol{P}(n-1)+b e(n) \boldsymbol{x}(n)\end{array}$ & $\alpha=1, \beta=0.001, a=0.98$, and $b=0.3$ \\
\hline Proposed algorithm & & $\begin{array}{l}K=4 . t=0.5 \text { for a white input and } t=0.35 \text { for } \\
\text { an } \operatorname{AR}(1) \text { process. }\end{array}$ \\
\hline
\end{tabular}

The misalignment results of the proposed algorithm and the competitors with a white input are compared in Fig. 1. It can be seen that, while the NLMS with $\mu=1$ exhibits the same convergence rate as the proposed algorithm, there is a margin of $26 \mathrm{~dB}$ in the steady-state misalignment of them. The SM-NLMS algorithm improves a little bit in the misalignment, but is still $23 \mathrm{~dB}$ worse than the proposed one. The NPVSS-NLMS, NVSS-NLMS, NVSS-LMS, $l_{2}$ VSS-LMS, and JO-NLMS algorithms show similar misalignments as the NLMS one with $\mu=0.055$, i.e., within $\pm 3 \mathrm{~dB}$. Among these algorithms, the NVSS-NLMS algorithm performs the best because of the fastest convergence and lowest misalignment. The proposed algorithm shows the same convergence rate as the NVSS-NLMS one while achieves a $9 \mathrm{~dB}$ improvement in the misalignment. It can also be found that the proposed algorithm achieves the closest performance to the miracle one, which denotes $\mu=1$ the theoretically best performance. To be specific, there is only a $4 \mathrm{~dB}$ margin between them after 50000 iterations, which is far less than the competitors. Moreover, it is worth to point out that the proposed algorithm has a further downward trend, which is hard to be found in the competitors.

The misalignment in the same condition as that in Fig. 1 is compared in Fig. 2, except that an $\operatorname{AR}(1)$ process is taken as the input of the identified system.
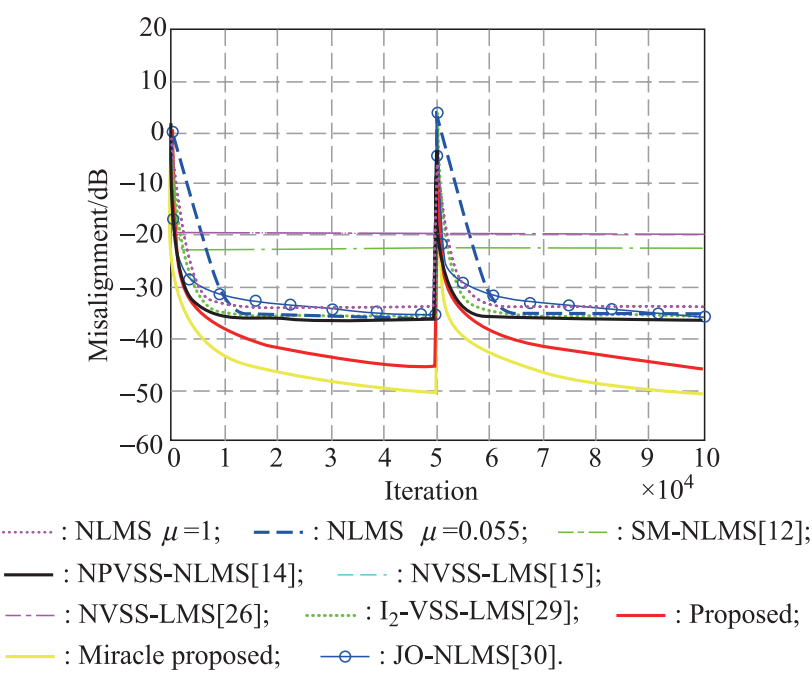

Fig. 1 Misalignment results of the NLMS with $\mu=1$ and 0.055 


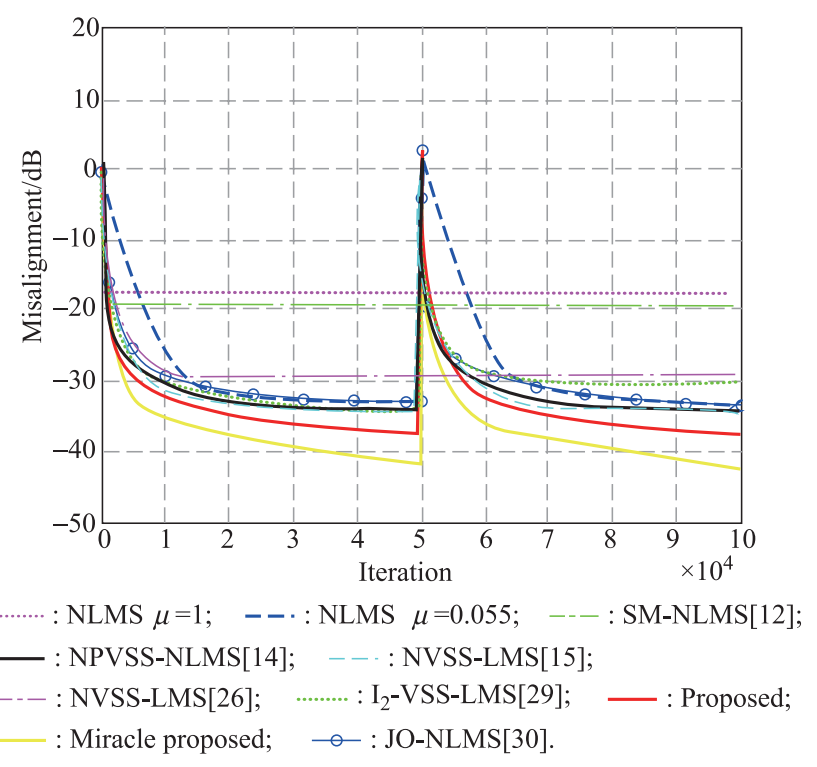

Fig. 2 Misalignment results of the NLMS with $\mu=1$ and 0.055

It can be seen that the performances of NPVSS-NLMS, NVSS-NLMS, and JO-NLMS algorithm become closer to each other compared with Fig. 1. Among the competitors, although the NVSS-NLMS algorithm still achieves the lowest misalignment, it shows an obvious compromise in the convergence rate, and thus can hardly be viewed as the best one. On the other hand, the proposed algorithm still exhibits the closest performance to the miracle one, i.e., outperforms all of the competitors, and the margin between them retains $4 \mathrm{~dB}$ as in Fig. 1, where the NLMS with $\mu=1$ and 0.055 , SM-NLMS [13], NPVSSNLMS [15], NVSS-NLMS [16], JO-NLMS [30], NVSSLMS [26], $l_{2}$-VSS-LMS [29], the proposed algorithm, and the miracle proposed algorithm. The input signal is a white Gaussian noise, the system changes at the 50 000th iteration, $L=128$, and $\mathrm{SNR}=20 \mathrm{~dB}$.

\section{Conclusions}

In this paper, a new NLMS adaptive filter with a variable regularization factor has been presented. The proposed algorithm is derived by incorporating the cost function of the conventional NLMS algorithm with the MD constraint weighted by a variable regularization factor. Analyses show that the step size and the regularization parameter in an NLMS algorithm can jointly be tuned by simply controlling the value of our variable regularization factor. It also demonstrates that such a factor can accelerate the convergence rate of the proposed algorithm. The optimal value of the factor is analyzed and then a method for determining it without any prior knowledge of the noise is given. While simulation results verify the effectiveness of the analytical ones, it also shows that the performance of the proposed algorithm is superior to the state-of-art algorithms.

\section{References}

[1] WIDROW B, STEARNS S D. Adaptive signal processing. Englewood Cliffs: Prentice-Hall, 1985.

[2] HAYKIN S. Adaptive filter theory. 4th ed. Upper Saddle Rier, New Jersey: Prentice-Hall, 2002.

[3] BENESTY J, HUANG Y. Adaptive signal processingapplications to real-world problems. Berlin: Springer-Verlag, 2003.

[4] HUANG Y, BENESTY J, CHEN J. Acoustic MIMO signal processing. Boston, MA: Springer, 2006.

[5] MA T, CHEN J, CHEN W, et al. Unsupervised robust adaptive filtering against impulsive noise. Journal of Systems Engineering and Electronics, 2012, 23(1): $32-39$.

[6] GUO L, JUNG L. Necessary and sufficient conditions for stability of LMS. IEEE Trans. on Automatic Control, 1995, 42(6): $761-770$.

[7] HASSIBI B, SAYED A H, KAILATH T. $\mathrm{H}_{\infty}$ optimality of the LMS algorithm. IEEE Trans. on Signal Processing, 1996, 44(2): $267-280$

[8] EVANS J B, XUE P, LIU B. Analysis and implementation of variable step size adaptive algorithms. IEEE Trans. on Signal Processing, 1993, 41(8): 2517-2535.

[9] ABOULNASR T, MAYYAS K. A robust variable step-size LMS-type algorithm: analysis and simulations. IEEE Trans. on Signal Processing, 1997, 45(3): 631-639.

[10] PAZAITIS D I, CONSTANTINIDES A G. A novel kurtosis driven variable step-size adaptive algorithm. IEEE Trans. on Signal Processing, 2002, 47(3): 864-872.

[11] ANTWEILER C, GRUNWALD J, QUACK H. Approximation of optimal step size control for acoustic echo cancellation. Proc. of the IEEE International Conference on Acoustics, 1997: $295-298$.

[12] MORGAN D R, KRATZER S G. On a class of computationally efficient, rapidly converging, generalized NLMS algorithms. IEEE Signal Processing Letters, 1996, 3(8): 245-247.

[13] GOLlamUdi S, NAGARAJ S, KAPOOR S, et al. Setmembership filtering and a set-membership normalized LMS algorithm with an adaptive step size. IEEE Signal Processing Letters, 1998, 5(5): 111-114.

[14] SHIN H C, SAYED A H, SONG W J. Variable step-size NLMS and affine projection algorithms. IEEE Signal Processing Letters, 2008, 11(2): $132-135$.

[15] BENESTY J, REY H, VEGA L R, et al. A nonparametric VSS NLMS algorithm. IEEE Signal Processing Letters, 2006, 13(10): $581-584$.

[16] HUANG H C, LEE J. A new variable step-size NLMS algorithm and its performance analysis. IEEE Trans. on Signal Processing, 2012, 60(4): 2055-2060.

[17] ARABLOUEI R, DOGANCAY K. Linearly-constrained recursive total least-squares algorithm. IEEE Signal Processing Letters, 2012, 19(12): $821-824$.

[18] BENESTY J, GAENSLER T, MORGAN D R, et al. Advances in network and acoustic echo cancellation. Berlin: SpringerVerlag, 2001.

[19] FAZA A, GRANT S L, BENESTY J. Adaptive regularization in frequency-domain NLMS filters. Proc. of the Signal Pro- 
cessing Conference, 2012: 2625-2628.

[20] BENESTY J, PALEOLOGU C, CIOCHINA S. On regularization in adaptive filtering. IEEE Trans. on Audio, Speech and Language Processing, 2011, 19(6): $1734-1742$.

[21] NI J, LI F. Variable regularisation parameter sign subband adaptive filter. Electronics Letters, 2010, 46(24): 1605-1607.

[22] MADER A, PUDER H, SCHMIDT G U. Step-size control for acoustic echo cancellation filters - an overview. Signal Processing, 2000, 80(9): $1697-1719$.

[23] SULYMAN A I, ZERGUINE A. Convergence and steady-state analysis of a variable step-size NLMS algorithm. Signal Processing, 2003, 83(6): 1255-1273.

[24] PARK P, CHANG M, KONG N. Scheduled-stepsize NLMS algorithm. IEEE Signal Processing Letters, 2009, 16(12): 1055 1058.

[25] SONG I, PARK P G. A normalized least-mean-square algorithm based on variable-step-size recursion with innovative input data. IEEE Signal Processing Letters, 2012, 19(12): $817-$ 820.

[26] YUAN Z, SONGTAO X. New LMS adaptive filtering algorithm with variable step size. Proc. of the International Conference on Vision, Image and Signal Processing, 2017: 1-4.

[27] LI M, XI X. A new variable step-size NLMS adaptive filtering algorithm. Proc. of the Information Technology and Applications, 2013: 236-239.

[28] HUAN Q Y, QIU X H, LIU X F. Variable step LMS algorithm using norm of the hyperbolic tangent function. Journal of Signal Processing, 2014, 30(1): 93 -99.

[29] WANG Y, SUN X, LIU L. A variable step size LMS adaptive filtering algorithm based on L2 norm. Proc. of the Signal Processing, Communications and Computing, 2016: 1-6.

[30] CIOCHINǍ S, PALEOLOGU C, BENESTY J. An optimized NLMS algorithm for system identification. Signal Processing, 2016, 118: $115-121$.

[31] BHOTTO M Z A, ANTONIOU A. New improved recursive least-squares adaptive-filtering algorithms. IEEE Trans. on Circuits and Systems I: Regular Papers, 2013, 60(6): 1548 1558.

[32] CASTOLDI F T, DE CAMPOS M L R. Application of a minimum-disturbance description to constrained adaptive filters. IEEE Signal Processing Letters, 2013, 20(12): 1215 1218.

[33] GUL M M U, MA X, LEE S. Timing and frequency synchronization for OFDM downlink transmissions using zadoff-chu sequences. IEEE Trans. on Wireless Communications, 2015, 14(3): 1716-1729.

\section{Biographies}

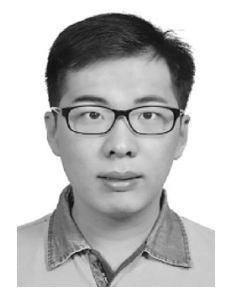

LI Zhoufan was born in 1992. He received his B.Sc. degree in electrical engineering from Fudan University, Shanghai, China, in 2015. He is currently working towards his M.S. degree in Department of Electronic Engineering, Fudan University, Shanghai, China. His research interests include digital signal processing and its application in wireless communication.

E-mail: 15210720031@fudan.edu.cn

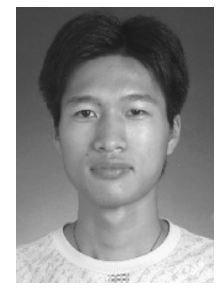

LI Dan was born in 1982. He received his B.Sc., M.S., and Ph.D. degrees in electrical engineering from Fudan University, Shanghai, China, in 2003, 2006, and 2013, respectively. Since 2006, he has been an lecturer at Department of Electronic Engineering, Fudan University, Shanghai, China. His research interests include digital signal processing and its application to nondestructive testing.

E-mail: lidan@fudan.edu.cn

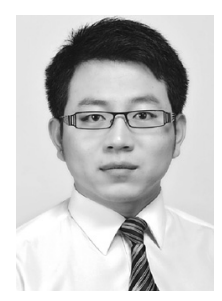

XU Xinlong was born in 1989 . He received his B.Sc. and M.S. degrees in electrical engineering from Fudan University, Shanghai, China, in 2011 and 2014, respectively. His research interests include signal processing and adaptive filtering. E-mail: 11210720032@fudan.edu.cn

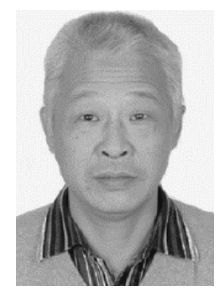

ZHANG Jianqiu was born in 1962 . He received his B.Sc. degree from East of China Institute of Engineering, Nanjing, and his M.S. and Ph.D. degrees from Harbin Institute of Technology (HIT), Harbin, China, in 1992 and 1996, respectively. He is currently a professor with the Department of Electronic Engineering, Fudan University, Shanghai, China. From 1999 to 2002, he was a senior research fellow at the School of Engineering, University of Greenwich, Chatham Maritime, U.K. In 1998, he was a visiting research scientist at the Institute of Intelligent Power Electronics, Helsinki University of Technology, Espoo, Finland. He was an associate professor from 1995 to 1997 and a lecturer from 1989 to 1994 in the Department of Electrical Engineering, HIT. During 1982 to 1987, he was an assistant electronic engineer at the 544th Factory, Hunan, China. His main research interests are signal processing and its application.

Email: jqzhang@ieee.org 